\title{
Efficacy and tolerability of oxycodone in moderate-severe cancer-related pain: A meta-analysis of randomized controlled trials
}

\author{
YU-MEI WANG ${ }^{1}$, ZU-WANG LIU $^{1}$, JIA-LI LIU ${ }^{3}$ and LEI ZHANG ${ }^{2}$ \\ Departments of ${ }^{1}$ Hospice, and ${ }^{2}$ Orthopedic Surgery, The Shengjing Affiliated Hospital of China Medical University, \\ Shenyang 110004; ${ }^{3}$ Department of Oncology, the First People's Hospital, Yueyang 414000, P.R. China
}

Received September 18, 2011; Accepted November 30, 2011

DOI: $10.3892 /$ etm.2012.571

\begin{abstract}
In order to evaluate the efficacy and tolerability of oxycodone in moderate-severe cancer-related pain, we conducted a systematic review of randomized controlled trials (RCTs). Publications addressing the efficacy and tolerability of oxycodone in moderate-severe cancer-related pain were selected from the Cochrane library, PubMed, Embase and CBM databases. Data were extracted from the studies by two independent reviewers. The meta-analysis was performed by RevMan 5.0.25 and STATA 9.2 software. From these data, odds ratios (ORs) or the standard mean difference (SMD) with $95 \%$ confidence intervals (CIs) were calculated. Finally, only seven RCTs were retrieved with a total of 613 cancer patients with moderate-severe pain. The meta-analysis results showed that oxycodone was statistically superior to other strong opioids based on pain intensity scores following intervention [weighted mean difference (WMD), $0.25 ; 95 \% \mathrm{CI}$, 0.05-0.45; P=0.01; WMD, -1.30; 95\% CI, -1.55-1.05; $\mathrm{P}<0.001$, respectively]. In addition, there were statistically significant differences between oxycodone and other strong opioids in cancer-related pain on the obvious effective rate and the overall effective rate (OR, 2.03; 95\% CI, 1.40-2.95; $\mathrm{P}=0.0002$; OR, 1.94; 95\% CI, 1.09-3.44; $\mathrm{P}=0.02$, respectively). Compared with other strong opioids, nausea and constipation occurred significantly less frequently with the use of oxycodone for cancer-related pain $(\mathrm{OR}=0.52,95 \% \mathrm{CI}=0.32-0.85, \mathrm{P}=0.009$; $\mathrm{OR}=0.55,95 \% \mathrm{CI}=0.35-0.87, \mathrm{P}=0.01$; respectively). In conclusion, this meta-analysis confirms that the efficacy and tolerability of oxycodone are superior to those of other strong
\end{abstract}

Correspondence to: Dr Yu-Mei Wang, Department of Hospice, the Shengjing Affiliated Hospital of China Medical University, Shenyang 110004, P.R. China

E-mail: cmu_wym@126.com

Key words: oxycodone, cancer-related pain, randomized controlled trial, meta-analysis opioids, including morphine sulfate, codeine and tramadol, supporting its use as an opioid for cancer-related pain.

\section{Introduction}

Cancer-related pain occurs in more than $80 \%$ of cancer patients prior to mortality (1). For patients with advanced cancer,pain was described as moderate-severe in approximately $40-50 \%$ and as very severe in $25-30 \%$ (2). Approximately $70 \%$ of patients with moderate-severe cancer-related pain require opioid analgesics during the course of the disease (3). Cancer-related pain may be managed with the various pharmacological and non-pharmacological methods currently available, but this is not always effective and numerous patients continue to suffer pain (4). Since the 1980s, treatment of cancer-related pain has been based on the World Health Organization (WHO) analgesic ladder. However, up to half of patients received inadequate analgesia and $30 \%$ of patients did not receive appropriate drugs for their pain (5). According to WHO guidelines, opioid analgesics are the mainstay of analgesic therapy and are classified according to their ability to control pain from mild, to mild-moderate, to moderate-severe intensity (6). Due to the intolerable adverse effects associated with opioids, approximately $20 \%$ of cancer patients may need to switch to an alternative opioid (7-9).

Morphine, oxycodone, methadone, hydromorphone, fentanyl, alfentanyl, buprenorphine, heroin, levorphanol and oxymorphone are the most widely used strong opioids for moderate-severe cancer-related pain in China. Oxycodone is a semisynthetic derivative of morphine. The efficacy and tolerability of oxycodone are similar to morphine, supporting its use as an opioid for moderate-severe cancer-related pain (10). It has been in clinical use since 1917, but patterns of use have differed worldwide, perhaps reflecting the lack of clinical studies investigating its efficacy (11). In the last ten years in China, the consumption of oxycodone has been increasing markedly. However, there is no evidence from high-quality comparative studies that oxycodone is superior to morphine and other opioids in terms of efficacy and tolerability (7). The aim of this study was to evaluate the efficacy and tolerability of oxycodone in moderate-severe cancer-related pain in China by conducting a meta-analysis from all eligible randomized controlled trials (RCTs) published to date. 


\section{Materials and methods}

Literature search. We performed an electronic search of the Cochrane library, PubMed, Embase and CBM databases to retrieve studies linking the efficacy and tolerability of oxycodone in moderate-severe cancer-related pain in China available up to August 2011 without language restrictions, using the following search tools: ('oxycodone', 'oxycodeinon', 'oxycone', 'dihydrohydroxycodeinone', 'pancodine' or 'oxycodone hydrochloride'), ('pain', 'ache' or 'aches'), ('neoplasms', 'cancer' or 'tumor'), ('therapeutics' or 'treatment') and ('randomized controlled trials', 'controlled clinical trials, randomized' or 'clinical trials, randomized'). The reference lists of major textbooks, reviews and included articles were identified through manual searches to find other potentially eligible studies. If more than one article was published by the same author using the same case series, we selected the research with the largest sample size.

Inclusion and exclusion criteria. In order to be eligible for inclusion in this meta-analysis, the following criteria were established: i) Clinical RCTs that addressed the efficacy and tolerability of oxycodone in moderate-severe cancer-related pain in China, ii) cancer patients with moderate-severe cancerrelated pain, iii) all routes of drug administration and all formulations of oxycodone were considered, and iv) studies that included sufficient genotype data for extraction. Studies were excluded when: i) They were not clinical RCTs that evaluated the efficacy and tolerability of the use of oxycodone for moderate-severe cancer-related pain in China, ii) they were meta-analyses, letters, reviews and editorial articles, iii) they were studies on combinations of oxycodone preparations (e.g., oxycodone and acetaminophen), iv) they were studies that were based on incomplete raw data and no usable data were reported, and v) they were duplicate publications.

Data extraction. Using a standardized form, data from published studies were extracted independently by two reviewers (Z.-W.L. and J.-L.L.) to populate the necessary information. From each of the included articles the following information was extracted: First author, year of publication, province, region, study design, intervention methods, number of cases, gender, age, quality evaluation, pain grade, pain intensity, pain relief and side-effects. For conflicting evaluations, an agreement was reached following a discussion with a third reviewer (Y.-M.W.).

Quality assessment of included studies. The quality of included studies was also independently assessed by two reviewers (Z.-W.L. and J.-L.L.), based on the Jadad score scale (11). This is a 5-point quality scale, with low-quality studies having a score of $\leq 2$ and high-quality studies a score of $\geq 3$. In addition, the categories or summary quality grades are defined as follows: Grade A, results are valid without marked major bias; grade B, study is susceptible to some bias that is unlikely to invalidate the results; and grade $\mathrm{C}$, significant bias is present that may invalidate the results (12-14). Any discrepancies between the two reviewers were resolved by discussion and consultation with a third reviewer (Y.-M.W.).

Statistical analysis. The meta-analysis was performed using the Review Manager (version 5.0.25; The Cochrane Collaboration; http://ims.cochrane.org/revman/download) and STATA package (version 10.0; Stata Corp., College Station, TX, USA). The differences of efficacy and tolerability between oxycodone and other strong opioids were estimated by odds ratios (ORs) or the standard mean difference (SMD) and 95\% confidence interval (95\% CI). Between-study heterogeneities were estimated using Cochran's $Q$ test $(12,15)$. We also quantified the effect of heterogeneity by the $\mathrm{I}^{2}$ test. $\mathrm{I}^{2}$ ranges between 0 and $100 \%$ and represent the proportion of inter-study variability that can be attributed to heterogeneity rather than chance. $\mathrm{I}^{2}$ values of 25,50 and $75 \%$ were defined as low, moderate and high estimates, respectively. When a significant $\mathrm{Q}$ test $(\mathrm{P}<0.10$ or $\mathrm{I}^{2}>50 \%$ ) indicated heterogeneity across studies, the random effects model was used for the meta-analysis, or else the fixed effects model was used (12). Publication bias was investigated by Begg's funnel plot, and funnel plot asymmetry was assessed by Egger's linear regression test (17). Statistical significance was considered when the P-value of Egger's test was $\leq 0.10$. All the $\mathrm{P}$-values were two-sided. To ensure the reliability and the accuracy of the results, two reviewers (Z.-W.L. and J.-L.L.) populated the data in the statistical software programs independently and obtained the same results.

\section{Results}

Characteristics of the included studies. The search strategy retrieved 37 potentially relevant studies. According to the inclusion criteria, only seven RCTs with the full-text available were included in this meta-analysis and 30 studies were excluded. The flow chart of the study selection is shown in Fig. 1. These seven RCTs included a total of 613 cancer patients with moderate-severe pain (18-24). All included studies were clinical observation studies, which evaluated the efficacy and tolerability of oxycodone for cancer-related pain in China. The publishing year of the included studies ranged from 2002 to 2010. The baseline characteristics and methodological quality of all included studies are shown in Table I.

Pain intensity scores. There were four studies reporting pain intensity score data that were included in this meta-analysis. Meta-analysis results showed that there was no significant difference between oxycodone and other strong opioids in pain intensity scores prior to intervention [weighted mean difference (WMD), 0.00; 95\% CI, -0.37-0.37; P=0.99]. However, oxycodone was statistically superior to other strong opioids with regard to pain intensity scores following intervention (WMD, 0.25; 95\% CI, 0.05-0.45; $\mathrm{P}=0.01$ ). Similarly, the meta-analysis of the four RCTs that had data on pain intensity difference showed results in favour of oxycodone (WMD, -1.30; 95\% CI, $-1.55-1.05 ; \mathrm{P}<0.001)$. As there was evidence of heterogeneity between the study estimates $\left(\mathrm{I}^{2}=55 \%, 91\right.$ and $99 \%$, respectively), the random effects model was used (Fig. 2).

Pain relief rate. The meta-analysis of all seven trials with pain relief rate data available showed that there was a statistically significant difference between oxycodone and other strong opioids for cancer-related pain on the obvious effective rate (OR, 2.03; 95\% CI, 1.40-2.95; $\mathrm{P}=0.0002$ ). In addition, the results also identified a significant difference between oxycodone and other strong opioids for cancer-related pain on the 


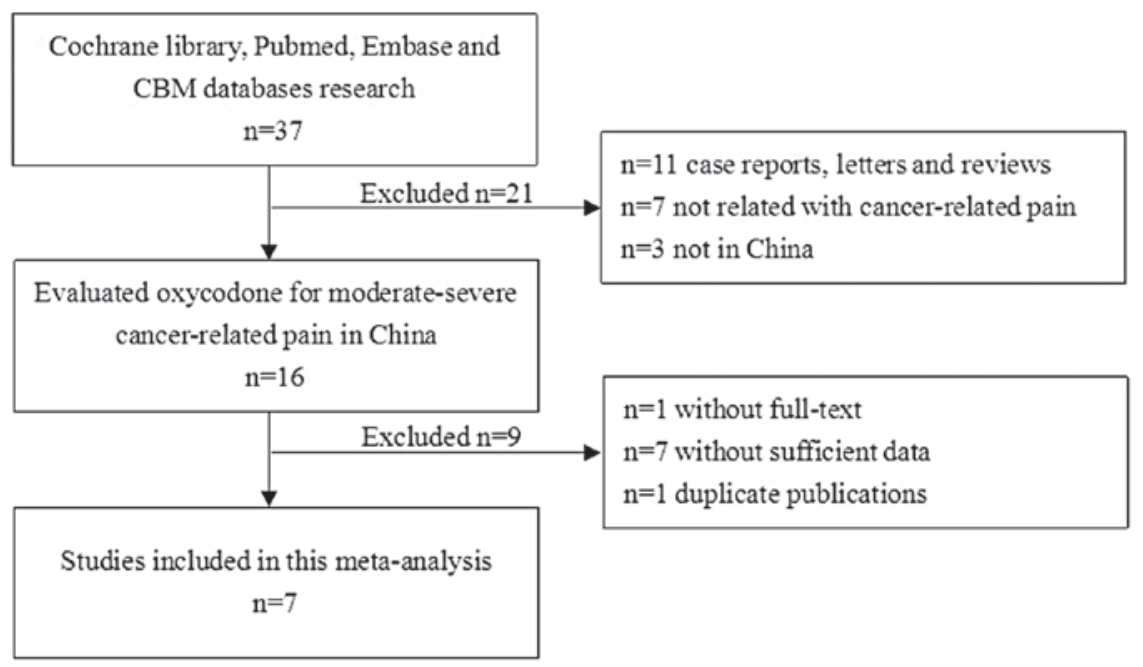

Figure 1. Flow chart showing study the selection procedure. Finally, only seven randomized controlled trials were retrieved with a total of 613 cancer patients with moderate-severe pain.

\begin{tabular}{|c|c|c|c|c|c|c|c|c|c|c|c|}
\hline \multirow{3}{*}{$\begin{array}{l}\text { Study or Subgroup } \\
\text { Before intervention }\end{array}$} & \multicolumn{3}{|c|}{ Observation } & \multicolumn{2}{|c|}{ Control } & \multirow{2}{*}{\multicolumn{3}{|c|}{$\begin{array}{c}\text { Std. Mean Difference } \\
\text { Iotal Weight } \quad \text { IV. Random. } 95 \% \mathrm{Cl}\end{array}$}} & \multicolumn{2}{|c|}{ Std. Mean Difference } & \\
\hline & \multirow[t]{2}{*}{ Mean } & \multicolumn{2}{|c|}{ SD Total } & Mean & SD & & & & \multicolumn{2}{|c|}{ IV. Random. $95 \% \mathrm{Cl}$} & \\
\hline & & & & & & & & & & \\
\hline Li et al 2002 & 6.61 & 1.24 & 18 & 7.47 & 1.01 & 17 & $18.0 \%$ & $-0.74[-1.43,-0.05]$ & & & \\
\hline Yu et al 2007 & 7 & 1.1 & 15 & 6.8 & 1.1 & 15 & $17.1 \%$ & $0.18[-0.54,0.89]$ & & - & \\
\hline Wang et al 2008 & 7.36 & 1.52 & 30 & 6.75 & 1.77 & 30 & $25.0 \%$ & $0.36[-0.15,0.88]$ & & $E=$ & \\
\hline Xiong et al 2008 & 6.57 & 1.83 & 142 & 6.5 & 1.77 & 141 & $39.9 \%$ & $0.04[-0.19,0.27]$ & & 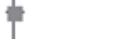 & \\
\hline Total $(95 \% \mathrm{Cl})$ & & & 205 & & & 203 & $100.0 \%$ & $0.00[-0.37,0.37]$ & & 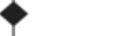 & \\
\hline \multicolumn{9}{|c|}{$\begin{array}{l}\text { Heterogeneity: } \mathrm{Tau}^{2}=0.08 ; \mathrm{Ch}^{2}=6.64, \text { df }=3(P=0.08) ; 1^{2}=55 \% \\
\text { Test for overall effect: } Z=0.02(P=0.99)\end{array}$} & & & \\
\hline \multicolumn{11}{|l|}{ After intervention } & \\
\hline Li et al 2002 & 2.17 & 2.38 & 18 & 3 & 2.03 & 17 & $8.8 \%$ & $-0.37[-1.03,0.30]$ & - & & \\
\hline Yu et al 2007 & 4 & 2.2 & 15 & 4.1 & 1.6 & 15 & $7.7 \%$ & $-0.05[-0.77,0.67]$ & & - & \\
\hline Wang et al 2008 & 1.85 & 1.34 & 30 & 3.34 & 1.67 & 30 & $13.7 \%$ & $-0.97[-1.51,-0.43]$ & $=$ & & \\
\hline Xiong et al 2008 & 5 & 1 & 142 & 4.4 & 0.97 & 141 & $69.7 \%$ & $0.61[0.37,0.85]$ & & $\mathbf{a}$ & \\
\hline Total $(95 \% \mathrm{Cl})$ & & & 205 & & & 203 & $100.0 \%$ & $0.25[0.05,0.45]$ & & $\bullet$ & \\
\hline \multicolumn{11}{|c|}{$\begin{array}{l}\text { Heterogeneity: } \mathrm{Chi}^{2}=32.43, \mathrm{df}=3(P<0.00001) ; \mathrm{I}^{2}=91 \% \\
\text { Test for overall effect: } Z=2.50(P=0.01)\end{array}$} & \\
\hline \multicolumn{11}{|l|}{ Difference } & \\
\hline Li et al 2002 & 4.44 & 1.93 & 18 & 4.47 & 2.18 & 17 & $14.1 \%$ & $-0.01[-0.68,0.65]$ & \multirow[b]{4}{*}{ 룰 } & - & \\
\hline Yu et al 2007 & 3 & 1.4 & 15 & 2.7 & 1.1 & 15 & $12.0 \%$ & $0.23[-0.49,0.95]$ & & & \\
\hline Wang et al 2008 & 5.51 & 1.1 & 30 & 3.41 & 1.67 & 30 & $18.7 \%$ & $1.47[0.89,2.04]$ & & $=$ & \\
\hline Xiong et al 2008 & 1.57 & 0.98 & 142 & 4.4 & 0.97 & 141 & $55.2 \%$ & $-2.89[-3.23,-2.56]$ & & & \\
\hline Total $(95 \% \mathrm{Cl})$ & & & 205 & & & 203 & $100.0 \%$ & $-1.30[-1.55,-1.05]$ & 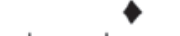 & & \\
\hline \multicolumn{9}{|c|}{$\begin{array}{l}\text { Heterogeneity: } \mathrm{Ch}^{2}=208.29, \mathrm{df}=3(P<0.00001) ; I^{2}=99 \% \\
\text { Test for overall effect: } Z=10.23(P<0.00001)\end{array}$} & $-4 \quad-2$ & $0 \quad 2$ & 4 \\
\hline
\end{tabular}

Figure 2. Differences in pain intensity scores between oxycodone and other strong opioids. CI, confidence interval.

overall effective rate (OR, 1.94; 95\% CI, 1.09-3.44; $\mathrm{P}=0.02)$ As there was no evidence of heterogeneity between the study estimates, the fixed effects model was used (Fig. 3).

Side-effects rate. A summary of the meta-analysis findings of the differences between oxycodone and other strong opioids for cancer-related pain is shown in Table II. Compared with other strong opioids, nausea and constipation occurred significantly less with the use of oxycodone for cancer-related pain
(OR, 0.52; 95\% CI, 0.32-0.85; P=0.009; OR, 0.55; 95\% CI, 0.35-0.87; $\mathrm{P}=0.01$, respectively).

There was no difference between oxycodone and other strong opioids in six other common side-effects: Dizziness, vomiting, sleepiness, pruritus, anorexia and dysuria (all $\mathrm{P}>0.05)$.

Publication bias. Publication bias of the literature was assessed based on pain relief rate data by Begg's funnel plot and Egger's 


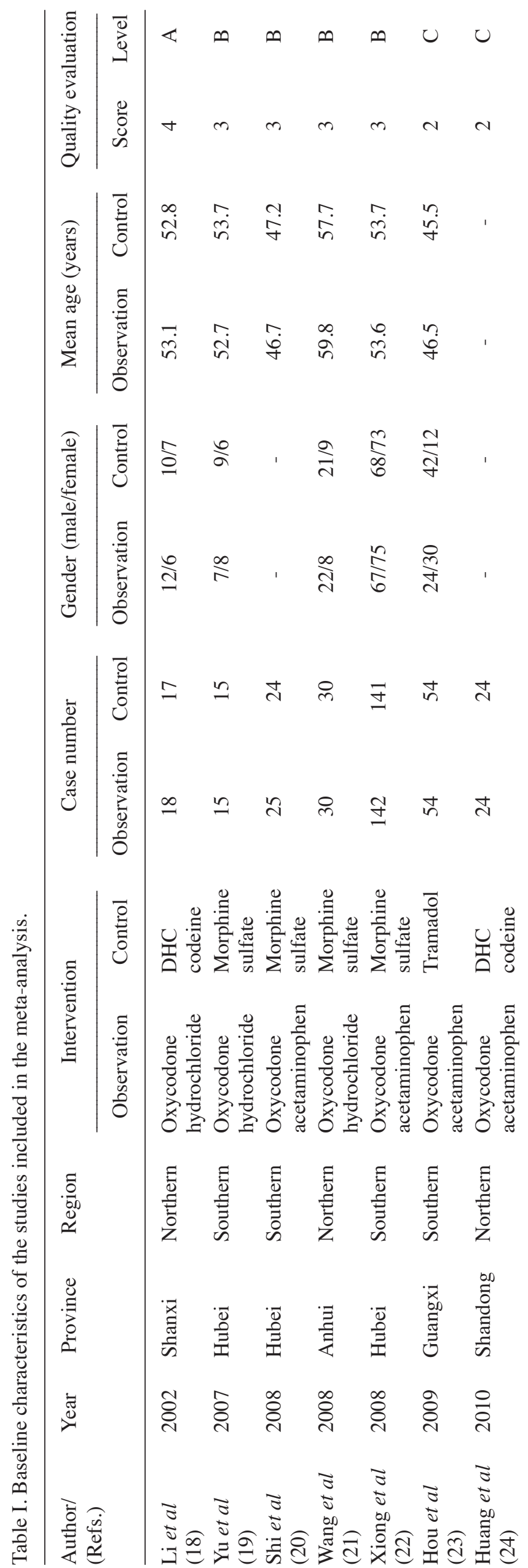

linear regression test. Egger's linear regression test was used to measure the asymmetry of the funnel plot. All graphical funnel plots of included studies appeared to be symmetrical (Fig. 4). Egger's test also showed that there was no statistical significance for all evaluations of publication bias $(\mathrm{P}=0.22$ and $\mathrm{P}=0.17$, respectively).

\section{Discussion}

Oxycodone is a semi-synthetic opioid that is effective in alleviating cancer-related pain, post-operative pain, osteoarthritis and neuropathic non-malignant pain (25). In 2001, oxycodone was suggested as one alternative to morphine in the WHO and the European Association for Palliative Care (EAPC) recommendations (26). During the past decade, oxycodone has still been considered as a step II opioid, or as a constituent of compound preparations with a non-opioid analgesic in China. However, the use of oxycodone has increased significantly in China (27). The clinical efficacy of oxycodone is similar to that of morphine sulfate, codeine and tramadol, but oxycodone is more potent with an equianalgesic ratio of 1/1.5-2 (28). Common side-effects of oxycodone are nausea, constipation, dizziness, vomiting, sleepiness, pruritus, anorexia and dysuria (29). In severe cases, intoxication coma, pulmonary edema and circulatory failure may appear and cause mortality (30). The aim of this study was to provide evidence-based guidelines on a wide variety of issues relevant to the use of oxycodone in cancer patients with moderate-severe cancer-related pain in China.

This meta-analysis was the first study to evaluate the differences between oxycodone and other strong opioids in moderate-severe cancer-related pain in China and may be able to add more information regarding this question. In this meta-analysis, we quantitatively assessed the differences in the efficacy and tolerability of oxycodone and other strong opioids in moderate-severe cancer-related pain in China. Finally, seven RCTs were included with a total of 613 cancer patients with moderate-severe pain. The meta-analysis results showed that oxycodone was statistically superior to other strong opioids in pain intensity scores following intervention and the differences between them were statistically significant. However, there was no significant difference between oxycodone and other strong opioids in pain intensity scores prior to intervention. In addition, there were statistically significant differences between oxycodone and other strong opioids for cancer-related pain on the obvious effective rate and overall effective rate. Compared with other strong opioids, nausea and constipation occurred significantly less with the use of oxycodone for cancer-related pain. There was no difference between oxycodone and other strong opioids in six other common sideeffects, including dizziness, vomiting, sleepiness, pruritus, anorexia and dysuria. Publication bias of the literature was assessed by Begg's funnel plot and Egger's linear regression test. No statistical significance for all evaluations of publication bias was found.

Similar to other meta-analyses, a number of limitations of this study should be addressed. First of all, a meta-analysis is a type of retrospective study and is limited by the quality of the primary studies. Secondly, although a perfect searching strategy was designed prior to initiating this study, and computerized and manual searching were performed simul- 
Table II. Meta-analysis of the differences in side-effect rates between oxycodone and other strong opioids for cancer-related pain.

\begin{tabular}{lcccccc}
\hline Side-effects & OR & $95 \%$ CI & P-value & \multicolumn{2}{c}{ Heterogeneity } & Effects model \\
\cline { 5 - 6 } & & & & $\mathrm{I}^{2}$ & P-value & \\
\hline Dizziness & 1.04 & $0.52-2.08$ & 0.90 & $0 \%$ & 0.83 & Fixed \\
Nausea & 0.52 & $0.32-0.85$ & 0.009 & $0 \%$ & 0.49 & Fixed \\
Vomiting & 0.61 & $0.33-1.13$ & 0.12 & $0 \%$ & 0.69 & Fixed \\
Sleepiness & 1.19 & $0.59-2.40$ & 0.62 & $0 \%$ & 0.61 & Fixed \\
Pruritus & 1.13 & $0.38-3.40$ & 0.82 & $0 \%$ & 0.73 & Fixed \\
Constipation & 0.55 & $0.35-0.87$ & 0.01 & $11 \%$ & 0.35 & Fixed \\
Anorexia & 0.70 & $0.14-3.51$ & 0.67 & $0 \%$ & 0.81 & Fixed \\
Dysuria & 1.45 & $0.43-4.91$ & 0.55 & $0 \%$ & 0.57 & Fixed \\
\hline
\end{tabular}

OR, odds ratio; CI, confidence interval.

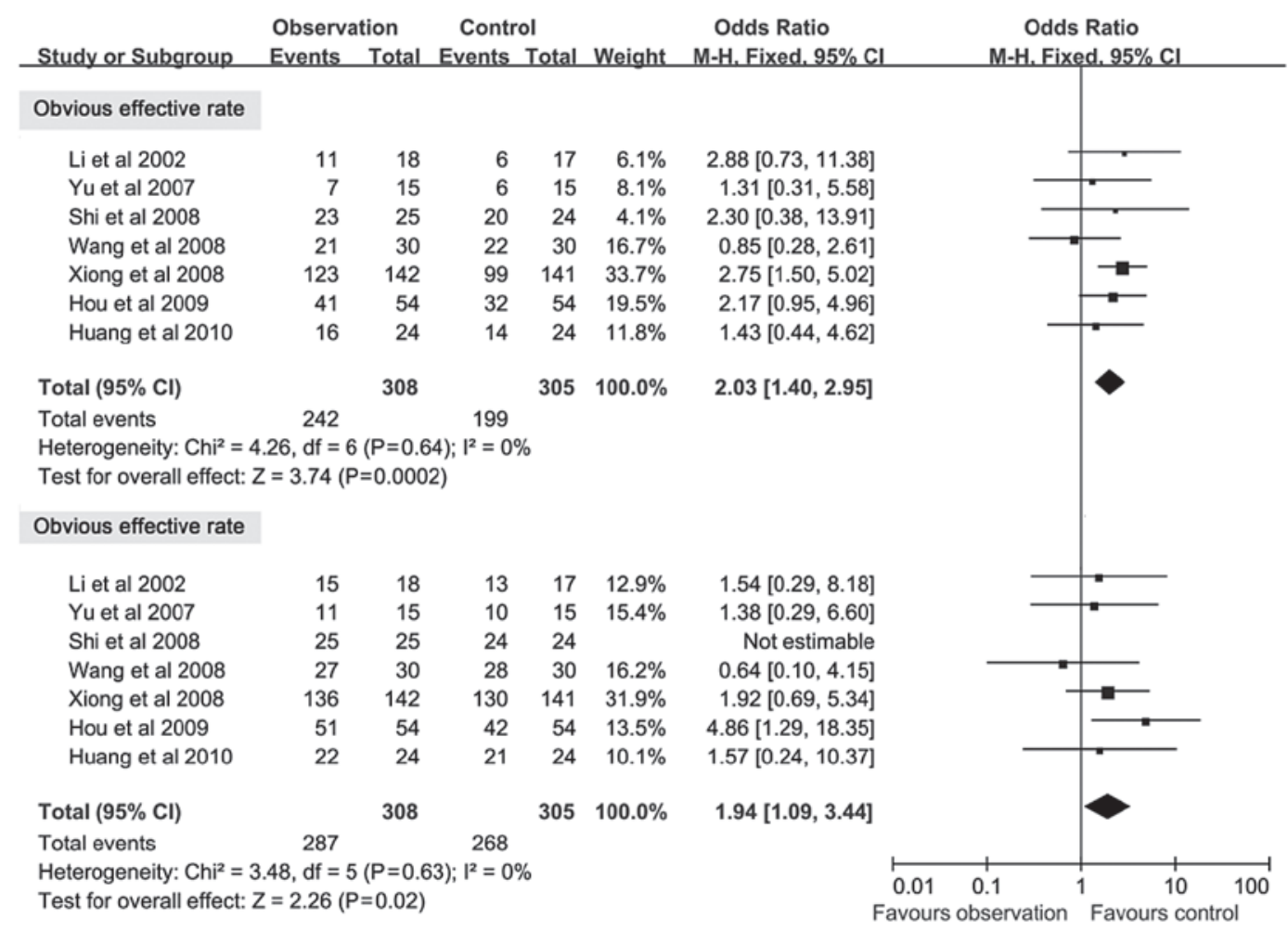

Figure 3. Differences in pain relief rate between oxycodone and other strong opioids. CI, confidence interval.
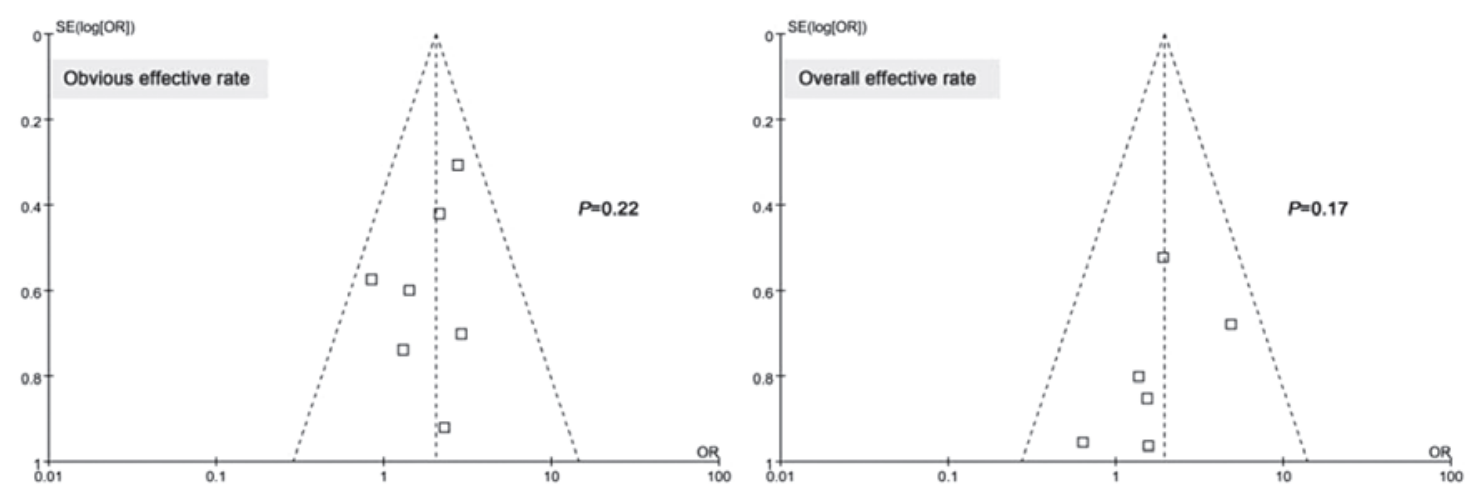

Figure 4. Publication bias of the literature. 
taneously, there is a possibility that suitable studies were not included. Thirdly, although we defined strict inclusion criteria, there may be potential studies that were not included in this meta-analysis due to incomplete raw data. Although we actively contacted the authors, they did not provide a comprehensive set of data. Most importantly, this meta-analysis was based on unadjusted data, and the main confounding variables were not available in the original papers and could also not be quantitatively analyzed.

In conclusion, our meta-analysis of seven RCTs demonstrated that the efficacy and tolerability of oxycodone are superior to other strong opioids, including morphine sulfate, codeine and tramadol, supporting its use as an opioid for cancer-related pain in China. As few studies are available in this field and current evidence remains limited, this conclusion should be further confirmed by large case-control studies with an adequate methodological quality and proper controlling for possible confounding factors.

\section{Acknowledgements}

We would like to thank Wu Yan (Department of Dermatology, the first Affiliated Hospital of China Medical University, Shenyang, P.R. China) for her valuable contribution and for kindly revising the manuscript.

\section{References}

1. Bruera E and Kim HN: Cancer pain. JAMA 290: 2476-2479, 2003.

2. Serlin RC, Mendoza TR, Nakamura Y, Edwards KR and Cleeland CS: When is cancer pain mild, moderate or severe? Grading pain severity by its interference with function. Pain 61: 277-284, 1995 .

3. Reid CM, Martin RM, Sterne JA, Davies AN and Hanks GW: Oxycodone for cancer-related pain: meta-analysis of randomized controlled trials. Arch Intern Med 166: 837-843, 2006.

4. Breivik H, Cherny N, Collett B, de Conno F, Filbet M, Foubert AJ, Cohen R and Dow L: Cancer-related pain: a pan-European survey of prevalence, treatment, and patient attitudes. Ann Oncol 20: $1420-1433,2009$

5. Gomes T, Juurlink DN, Dhalla IA, Mailis-Gagnon A, Paterson JM and Mamdani M: Trends in opioid use and dosing in the socioeconomically disadvantaged. Open Med 5: E13-E22, 2011.

6. Garcia del Pozo J, Carvajal A, Viloria JM, Velasco A and Garcia del Pozo V: Trends in the consumption of opioid analgesics in Spain. Higher increases as fentanyl replaces morphine. Eur J Clin Pharmacol 64: 411-415, 2008.

7. Jost L and Roila F; ESMO Guidelines Working Group: Management of cancer pain: ESMO Clinical Practice Guidelines. Ann Oncol (Suppl 5): v257-v260, 2010.

8. Panchal SJ, Müller-Schwefe P and Wurzelmann JI: Opioidinduced bowel dysfunction: prevalence, pathophysiology and burden. Int J Clin Pract 61: 1181-1187, 2007.

9. Paulozzi LJ and Ryan GW: Opioid analgesics and rates of fatal drug poisoning in the United States. Am J Prev Med 31: 506-511, 2006.

10. Boudreau D, Von Korff M, Rutter CM, Saunders K, Ray GT, Sullivan MD, Campbell CI, Merrill JO, Silverberg MJ, Banta-Green C and Weisner C: Trends in long-term opioid therapy for chronic non-cancer pain. Pharmacoepidemiol Drug Saf 18: 1166-1175, 2009.
11. Jadad AR, Moore RA, Carroll D, Jenkinson C, Reynolds DJ, Gavaghan DJ and McQuay HJ: Assessing the quality of reports of randomized clinical trials: is blinding necessary? Control Clin Trials 17: 1-12, 1996.

12. Lubin JH and Gail MH: Biased selection of controls for case-control analyses of cohort studies. Biometrics 40: 63-75, 1984.

13. Kjaergard LL, Villumsen J and Gluud C: Reported methodologic quality and discrepancies between large and small randomized trials in meta-analyses. Ann Intern Med 135: 982-989, 2001.

14. Guyatt G, Gutterman D, Baumann MH, Addrizzo-Harris D, Hylek EM, Phillips B, Raskob G, Lewis SZ and Schünemann H: Grading strength of recommendations and quality of evidence in clinical guidelines: Report from an american college of chest physicians task force. Chest 129: 174-181, 2006.

15. Park-Wyllie LY, Mamdani MM, Li P, Gill SS, Laupacis A and Juurlink DN: Cholinesterase inhibitors and hospitalization for bradycardia: a population-based study. PLoS Med 6: e1000157, 2009.

16. Charlson ME, Pompei P, Ales KL and MacKenzie CR: A new method of classifying prognostic comorbidity in longitudinal studies: development and validation. J Chronic Dis 40: 373-383, 1987.

17. Mamdani M, Sykora K, Li P, Normand SL, Streiner DL, Austin PC, Rochon PA and Anderson GM: Reader's guide to critical appraisal of cohort studies: 2. Assessing potential for confounding. BMJ 330: 960-962, 2005.

18. Li SY, Shang ZZ and Xue G: Clinical efficacy of oxycodone hydrochloride controlled-release tablets for cancer pain. Shanxi Oncol Med 10: 16-18, 2002.

19. Yu XH, Wu DG, Yang JF and Gao M: Clinical efficacy of oxycodone hydrochloride controlled-release tablets in management of moderate and severe cancer pain. Chin J Hosp Pharm 27: 1277-1278, 2007.

20. Shi L, Xiong HH, Yang L and Yu SY: Analgesic effect of oxycodone-acetaminophen tablets in patients with cervical cancer. Chin J New Drugs 17: 1884-1885, 2008.

21. Wang P, Liu Y, Wang T and Chen BZ: The observation of curative effect of oxycodone hydrochloride controlled-release fablets in management of moderate and severe cancer pain. Mod Oncol 16: 1983-1985, 2008.

22. Xiong J, Liang C, Wu X, Zhao YX, Fu L and Zhou YF: Efficacy and safety of oxycodone-acetaminophen tablet in 142 patients with different types of moderate and advanced cancer pain. Chin J New Drugs 17: 1877-1900, 2008.

23. Hou EC and Wang X: Clinical efficacy of oxycodone-acetaminophen tablet in management of cancer pain and its effect on immune function. J Guangxi Med Univ 26: 446-448, 2009.

24. Huang SW, Xie YF and Dong J: The observation of clinical eficacy of oxycodone-acetaminophen tablet in management of digestive cancer pain. Mod J Integr Tradit Chin West Med 19: 2628-2629, 2010.

25. Riley J, Eisenberg E, Müller-Schwefe G, Drewes AM and Arendt-Nielsen L: Oxycodone: a review of its use in the management of pain. Curr Med Res Opin 24: 175-192, 2008.

26. King SJ, Reid C, Forbes K and Hanks G: A systematic review of oxycodone in the management of cancer pain. Palliat Med 25: 454-470, 2011.

27. Pan H, Zhang Z, Zhang Y, Xu N, Lu L, Dou C, Guo Y, Wu S, Yue J, Wu D and Dai Y: Efficacy and tolerability of oxycodone hydrochloride controlled-release tablets in moderate to severe cancer pain. Clin Drug Investig 27: 259-267, 2007.

28. Ordóñez Gallego A, González Barón M and Espinosa Arranz E: Oxycodone: a pharmacological and clinical review. Clin Transl Oncol 9: 298-307, 2007.

29. Eisenberg E, McNicol ED and Carr DB: Efficacy and safety of opioid agonists in the treatment of neuropathic pain of nonmalignant origin: systematic review and meta-analysis of randomized controlled trials. JAMA 293: 3043-3052, 2005.

30. Leppert W: Role of oxycodone and oxycodone/naloxone in cancer pain management. Pharmacol Rep 62: 578-591, 2010. 